\title{
Feasibility of anthropometric indices to identify dyslipidemia among adults in Jilin Province: a cross-sectional study
}

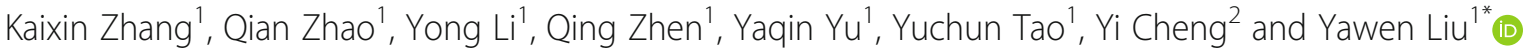

\begin{abstract}
Background: Dyslipidemia and other cardiovascular disease (CVD) risk factors have a strong association with obesity. Anthropometric indices have been widely used to evaluate obesity in clinical and epidemiological studies. We aim to investigate association between serum lipid levels and different anthropometric indices.

Methods: Our study included 17,554 participants. We mainly investigated area under the receiver operating characteristic (AUROC) curves and optimal operating points (OOPs) between the anthropometric indices and serum lipid levels or categories of abnormal serum lipid indices.

Results: For predicting one/two categories of abnormal serum lipid indices among the anthropometric indices, AUROC value of WC was the highest in men (0.718), and AUROC values of BRI and WHtR were the highest in women (0.700 and 0.700) (all $P<0.001$ ); OOP of WC was 82.450 in men; OOPs of BRI and WHtR were 3.435 and 0.504 in women. For predicting three/more categories of abnormal serum lipid indices among the anthropometric indices, AUROC value of WC was the highest in men (0.806), and AUROC values of BRI and WHtR were the highest in women (0.783 and 0. 783) (all $P<0.001$ ); OOP of WC was 84.150 in men; OOPs of BRI and WHtR were 3.926 and 0.529 in women.

Conclusions: WC was a good predictor for one/two or three/more categories of abnormal serum lipid indices in men. However, BRI and WHtR were good predictors for one/two or three/more categories of abnormal serum lipid indices in women. ABSI showed the weakest predictive power.
\end{abstract}

Keywords: Dyslipidemia, Anthropometric indices, Serum lipid indices

\section{Background}

Dyslipidemia is a critical risk-factor of cardiovascular disease (CVD) [1], contributing to mortality in patients with CVD [2, 3]. Dyslipidemia is defined by abnormal levels of total cholesterol (TC), or low-density cholesterol (LDL-C), or high-density cholesterol (HDL-C), or triglycerides (TGs) individually or in combination, which are based on biochemical measurement [1]. Although limited cost is required for the measurement of TC, TG, LDL-C, and HDL-C for one person, financial consumption on the basis of large population has brought huge socioeconomic burden to a nation. For this reason, dyslipidemia costs $\$ 16$ billion every year in China [4]. Screening

\footnotetext{
* Correspondence: ywliu@jlu.edu.cn

${ }^{1}$ Department of Epidemiology and Biostatistics, School of Public Health, Jilin University, Changchun 130021, China

Full list of author information is available at the end of the article
}

population at high risk of dyslipidemia, using a simple, convenient, and cost-effective way, rather than measuring TG, TC, LDL-C, and HDL-C, is necessary for controlling CVD.

Dyslipidemia and other CVD risk factors (hypertension and diabetes) have a strong association with obesity [5]. Anthropometric indices have been widely used to evaluate obesity in clinical and epidemiological studies [6], because such measurements only require height weight scales and tapes that are robust and inexpensive.

Body mass index (BMI) reflects the overall distribution of body fat [7], which is a widely accepted anthropometric index of overweight and obesity [8]. Risk of CVD and/or Metabolic syndrome (MetS) increases with increasing of BMI [9]. However, compared with BMI, waist-to-hip ratio (WHR) and waist circumference (WC) can better reflect the accumulation of intra-abdominal 
fat [10-12]. WHR has also been proposed as a predictor of CVD risk factors among Tehranian adult men [12]. WHR has the weakest association with CVD risks among WC, BMI, and waist-to-height ratio (WHtR) [13], largely because nonobese individuals theoretically have the same WHR as obese ones in that WHR can remain constant with changes in weight [14]. Notably, WC better describes abdominal shape than BMI or WHR, and is highly associated with CVD risk factors, especially diabetes [15-17].

WHtR has been proposed as an indicator of abdominal obesity to evaluate variations of body size [18-20]. Several studies on Asian and Caucasian populations have found WHtR is superior to WC in identifying cases with CVD risk factors [12, 18, 21-24]. Furthermore, metaanalyses support those findings $[25,26]$.

New anthropometric indices, such as a body shape index (ABSI) and body roundness index (BRI) has been proposed recently. ABSI has been found to be more correlated with mortality rate than BMI or WC [27-29]. BRI predicts the percentage of body fat, evaluating health status. Up to date, only a few studies have investigated whether ABSI and BRI are suitable predictors for identifying CVD and/or MetS risk factors [30, 31].

It is still unclear which anthropometric index (BMI, WC, WHR, WHtR, ABSI, and BRI) could be the most appropriate predictor for identifying dyslipidemia in northeast China. In this study, we investigated association between serum lipid levels and the anthropometric indices in participants from Jilin Province to explore predictive ability of those indices for dyslipidemia.

\section{Methods}

\section{Study population}

The data in this study were collected from a crosssectional survey of chronic diseases and related risk

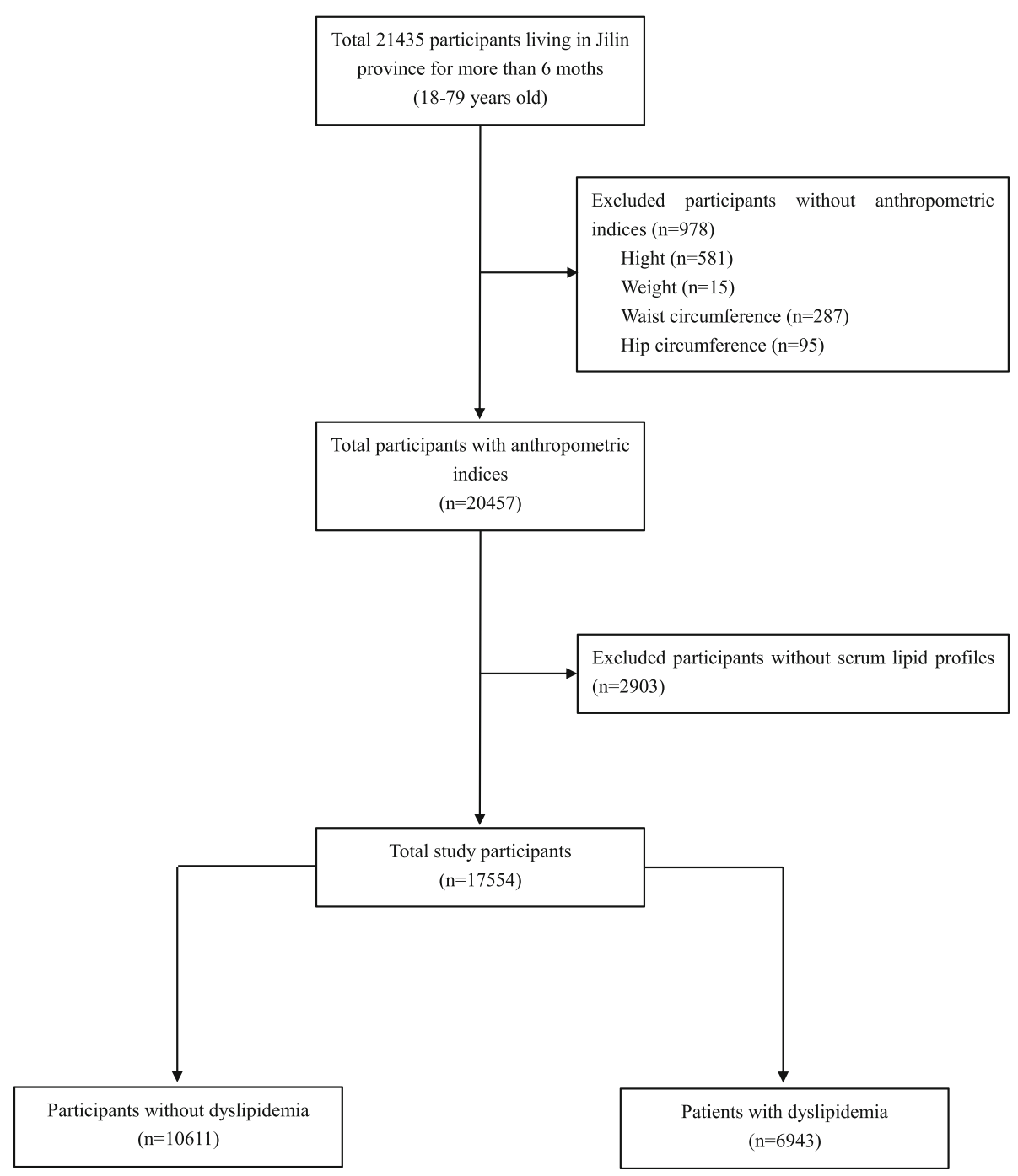

Fig. 1 Flow chart for this investigation 
factors among adults in Jilin Province, China in 2012. The survey used a multistage cluster random sampling design to select a representative sample population aged from 18 to 79 years old who had lived in Jilin Province for more than 6 months in nine different cities/prefecture in Jilin Province, including Changchun, Jilin, Siping, Liaoyuan, Tonghua, Baishan, Songyuan, Baicheng, and Yanbian Korean Autonomous Prefecture. The survey involved 21,435 participants. Questionnaires, data of anthropometric measurements, and data of fasting blood tests were collected from each participant. Demographic characteristics (sex, age, and nationality) were collected from the questionnaires. The anthropometric data (height, weight, WC, and hip circumference) were got using height weight scales and tapes. Fasting blood tests were performed after fasting at least $12 \mathrm{~h}$. Finally, this study included 17,554 participants with complete sets of data (Fig. 1).

\section{Sampling method}

We selected participants using multistage hierarchical random cluster sampling method. Firstly, according to the ratio of population, location, and level of economic development, we selected 32 representative districts/counties from the nine cities/prefecture of Jilin Province. Secondly, we randomly selected three or four towns from each of the selected district/ county using probability proportionate to size sampling. Thirdly, we randomly selected three administrative villages from each of the selected town using probability proportionate to size sampling. Fourthly, we selected one sub-village from each of the selected administrative village using simple random sampling. Finally, we randomly selected an adult aged 18 to 79 years old from every household in the each selected sub-village.

\section{Calculation of anthropometric indices}

WHR, WHtR [7, 32], BMI [33], ABSI [27], and BRI [28] were calculated using the following formulas.

$$
\begin{aligned}
& \mathrm{WHR}=\mathrm{WC}(\mathrm{cm}) / \mathrm{HC}(\mathrm{cm}) \\
& \mathrm{WHtR}=\mathrm{WC}(\mathrm{cm}) / \text { height }(\mathrm{cm}) \\
& \mathrm{BMI}=\text { Weight }(\mathrm{kg}) / \text { Height }\left(\mathrm{m}^{2}\right) \\
& \mathrm{ABSI}=\frac{\mathrm{Wc}}{B M I^{2 / 3} \text { height }^{1 / 2}} \\
& B R I=364.2-365.5 \times \sqrt{1-\left(\frac{(w c /(2 \pi))^{2}}{(0.5 \text { height })^{2}}\right)}
\end{aligned}
$$

\section{Laboratory assay}

Fasting serum samples were used to measure TG, TC, LDL-C, and HDL-C using MODULE P800 biochemical analysis machine (Roche Co., Ltd. Shanghai, China).

Dyslipidemia was defined according to the Chinese guidelines on the prevention and treatment of dyslipidemia in adults (2007) [34]: TG $\geq 2.26 \mathrm{mmol} / \mathrm{L}(200 \mathrm{mg} /$ $\mathrm{dL})$ as high; $\mathrm{TC} \geq 6.22 \mathrm{mmol} / \mathrm{L}(240 \mathrm{mg} / \mathrm{dL})$ as high; $\mathrm{LDL}-\mathrm{C} \geq 4.14 \mathrm{mmol} / \mathrm{L}(160 \mathrm{mg} / \mathrm{dL})$ as high; and HDL-C $<1.04 \mathrm{mmol} / \mathrm{L}(40 \mathrm{mg} / \mathrm{dL})$ as low.

\section{Statistical analysis}

All statistical analyses were performed using SPSS version 21.0 (SPSS Inc., Chicago, IL, USA). Mean and standard deviation were used to express normally distributed continuous variables, and median and Q1 to Q3 were used to express abnormally distributed continuous variables, with Q1 being the 25th percentile and Q3 being the 75th percentile. Continuous variables were tested for normality using the Kolmogorov-Smirnov test. Comparisons between men and women were performed using two independent samples t-test or Mann-Whitney $\mathrm{U}$ test for continuous variables. Relationship between the anthropometric indices and serum lipid levels was analyzed using Spearman's correlation analysis and Partial correlation analysis. Receiver operating characteristic (ROC) analysis was used to calculate the area under ROC curves (AUROC) between the anthropometric indices and serum lipid levels. Considering that the optimal cut-off point balance sensitivity (SEN) and specificity (SPE) of anthropometric index, we used optimal operating point (OOP) which is the maximum Youden index (SEN + SPE -1) [35] to select the optimal cut-off point to predict dyslipidemia. $P<0.05$ was considered statistically significant.

\section{Results}

Baseline characteristics of the participants

Baseline characteristics of the participants stratified by gender are shown in Table 1. A total of 17,554 participants, including 10,611 normal people $(60.448 \%)$ and 6943 patients with dyslipidemia (39.552\%), were composed of 8080 men (46.029\%) and 9474 women (53.971\%). The median age of the participants was 48 years old in both men and women. For anthropometric indices, the medians of height, weight, hip circumference, WC, BMI, ABSI, and WHR were higher, but those of BRI and WHtR were lower in men (all $P<0.001$ ). For serum lipid levels, the medians of TC level were similar $(P=0.121)$, LDL-C level $(P<0.001)$ and HDL-C level $(P<0.001)$ were higher, but TG level $(P<0.001)$ was lower in women. 
Table 1 Descriptive baseline characteristics of the participants

\begin{tabular}{|c|c|c|c|c|}
\hline & Men(8080) & Women(9474) & $t / Z$ & $P$ \\
\hline Age (years) & $48(37,57)$ & $48(40,58)$ & -6.007 & $<0.001$ \\
\hline Height (cm) & $169.200(165.000,173.800)$ & $157.500(153.500,161.300)$ & -93.614 & $<0.001$ \\
\hline Weight (kg) & $68.900(61.200,77.175)$ & $59.200(53.100,65.900)$ & -54.837 & $<0.001$ \\
\hline $\mathrm{HC}(\mathrm{cm})$ & $95.200(90.600,100.000)$ & $94.000(90.000,99.000)$ & -8.811 & $<0.001$ \\
\hline WC $(\mathrm{cm})$ & $84.800(77.000,92.000)$ & $80.000(73.000,87.200)$ & -26.242 & $<0.001$ \\
\hline BMI (kg/m2) & 24.152(21.719,26.680) & $23.936(21.586,26.469)$ & -2.732 & $<0.001$ \\
\hline ABSI (m11/6 kg-2/3) & $0.078(0.075,0.081)$ & $0.077(0.073,0.080)$ & -13.303 & $<0.001$ \\
\hline $\mathrm{BRI}$ & $3.385(2.594,4.187)$ & $3.540(2.669,4.511)$ & -9.481 & $<0.001$ \\
\hline WHR & $0.885 \pm 0.669$ & $0.849 \pm 0.073$ & 34.087 & $<0.001$ \\
\hline $\mathrm{WH} \mathrm{tR}$ & $0.501 \pm 0.061$ & $0.510(0.462,0.558)$ & -9.481 & $<0.001$ \\
\hline $\mathrm{TG}(\mathrm{mmol} / \mathrm{L})$ & $1.570(1.040,2.540)$ & $1.405(0.960,2.140)$ & -12.645 & $<0.001$ \\
\hline $\mathrm{TC}(\mathrm{mmol} / \mathrm{L})$ & $4.770(4.160,5.460)$ & $4.790(4.140,5.533)$ & -1.552 & 0.121 \\
\hline $\mathrm{LDL}-\mathrm{C}(\mathrm{mmol} / \mathrm{L})$ & $2.830(2.310,3.398)$ & $2.870(2.330,3.510)$ & -4.163 & $<0.001$ \\
\hline $\mathrm{HDL}-\mathrm{C}(\mathrm{mmol} / \mathrm{L})$ & $1.280(1.070,1.550)$ & $1.380(1.160,1.640)$ & -16.211 & $<0.001$ \\
\hline
\end{tabular}

HC Hip Circumference, WC Waist Circumference, BMI Body Mass Index, ABSI A Body Shape Index, BRI Body Roundness Index, WHR Waist-to-Hip Ratio, WHtR Waist-to-Height Ratio, TG Triglyceride, TC Total Cholesterol, LDL-C Low Density Lipoprotein Cholesterol, HDL-C High Density Lipoprotein Cholesterol

Table 2 Spearman correlation coefficient between anthropometric indices and serum lipid levels

\begin{tabular}{|c|c|c|c|c|c|c|c|c|}
\hline & \multicolumn{2}{|c|}{$\mathrm{TG}$} & \multicolumn{2}{|c|}{$\mathrm{TC}$} & \multicolumn{2}{|c|}{ LDL-C } & \multicolumn{2}{|c|}{$\mathrm{HDL}-\mathrm{C}$} \\
\hline & $r$ & $P$ & $r$ & $P$ & $r$ & $P$ & $r$ & $P$ \\
\hline \multicolumn{9}{|l|}{ Total } \\
\hline WC & 0.471 & $<0.001$ & 0.261 & $<0.001$ & 0.248 & $<0.001$ & -0.360 & $<0.001$ \\
\hline BMI & 0.419 & $<0.001$ & 0.218 & $<0.001$ & 0.217 & $<0.001$ & -0.341 & $<0.001$ \\
\hline$A B S I$ & 0.291 & $<0.001$ & 0.231 & $<0.001$ & 0.204 & $<0.001$ & -0.156 & $<0.001$ \\
\hline BRI & 0.458 & $<0.001$ & 0.293 & $<0.001$ & 0.281 & $<0.001$ & -0.316 & $<0.001$ \\
\hline WHR & 0.452 & $<0.001$ & 0.269 & $<0.001$ & 0.233 & $<0.001$ & -0.306 & $<0.001$ \\
\hline WHtR & 0.458 & $<0.001$ & 0.293 & $<0.001$ & 0.281 & $<0.001$ & -0.316 & $<0.001$ \\
\hline \multicolumn{9}{|l|}{ Men } \\
\hline WC & 0.475 & $<0.001$ & 0.249 & $<0.001$ & 0.213 & $<0.001$ & -0.399 & $<0.001$ \\
\hline BMI & 0.461 & $<0.001$ & 0.224 & $<0.001$ & 0.201 & $<0.001$ & -0.406 & $<0.001$ \\
\hline$A B S I$ & 0.231 & $<0.001$ & 0.187 & $<0.001$ & 0.139 & $<0.001$ & -0.140 & $<0.001$ \\
\hline $\mathrm{BRI}$ & 0.468 & $<0.001$ & 0.270 & $<0.001$ & 0.227 & $<0.001$ & -0.375 & $<0.001$ \\
\hline WHR & 0.443 & $<0.001$ & 0.261 & $<0.001$ & 0.197 & $<0.001$ & -0.316 & $<0.001$ \\
\hline WHtR & 0.468 & $<0.001$ & 0.270 & $<0.001$ & 0.227 & $<0.001$ & -0.375 & $<0.001$ \\
\hline \multicolumn{9}{|l|}{ Women } \\
\hline WC & 0.455 & $<0.001$ & 0.286 & $<0.001$ & 0.300 & $<0.001$ & -0.303 & $<0.001$ \\
\hline BMI & 0.383 & $<0.001$ & 0.214 & $<0.001$ & 0.232 & $<0.001$ & -0.283 & $<0.001$ \\
\hline ABSI & 0.329 & $<0.001$ & 0.267 & $<0.001$ & 0.260 & $<0.001$ & -0.152 & $<0.001$ \\
\hline BRI & 0.469 & $<0.001$ & 0.311 & $<0.001$ & 0.320 & $<0.001$ & -0.291 & $<0.001$ \\
\hline WHR & 0.450 & $<0.001$ & 0.299 & $<0.001$ & 0.295 & $<0.001$ & -0.270 & $<0.001$ \\
\hline $\mathrm{WH} \mathrm{tR}$ & 0.469 & $<0.001$ & 0.311 & $<0.001$ & 0.320 & $<0.001$ & -0.291 & $<0.001$ \\
\hline
\end{tabular}




\section{Relationship between anthropometric indices and serum lipid levels}

We investigated the relationship between anthropometric indices and serum lipid levels using Spearman's rank test (Table 2). For men, high correlations were identified between WC and TG level $(r=0.475, P<0.001)$, between the levels of BRI and WHtR and TC level (both $r=0.270, P<0.001$ ), between the levels of BRI and WHtR and LDL-C level (both $r=0.227, P<0.001$ ), and between BMI and HDL-C level $(r=-0.406, P<$ 0.001 ). For women, high correlations were identified between the levels of BRI and WHtR and TG level (both $r=0.469, P<0.001$ ), between the levels of BRI and WHtR and TC level (both $r=0.311, P<0.001$ ), between the levels of BRI and WHtR and LDL-C level (both $r=0.320, P<0.001$ ), and between WC and HDL$C$ level $(r=-0.303, P<0.001)$. After adjusting for age, for men, high correlations were identified between WHtR and the levels of TG, TC, and LDL-C $(r=0.326$, 0.232 , and 0.187 , respectively; all $P<0.001$ ), and between WC and HDL-C level $(r=-0.376, P<0.001)$. For women, high correlations were identified between WC and the levels of TG, TC, and LDL-C $(r=0.231,0.131$, and 0.154 , respectively; all $P<0.001)$, and between WC and HDL-C level $(r=-0.282, P<0.001)$ (Table 3$)$.

\section{Relationship between anthropometric indices and categories of abnormal serum lipid indices}

Because there are no reference and guideline to reveal relationship between anthropometric indices and categories of abnormal serum lipid indices, and there are few patients with three categories of abnormal serum lipid indices and four categories of abnormal serum lipid indices, we further classified the patients into two groups: patients with one/two categories of abnormal serum lipid indices (group 1) and patients with three/ more categories of abnormal serum lipid indices (group 2 ). We investigated the relationship between anthropometric indices and categories of abnormal serum lipid indices using Spearman rank test (Table 4). For men, high correlation was identified between WC and categories of abnormal serum lipid indices $(r=0.383, P<$ 0.001 ). For women, high correlations were identified between the levels of BRI and WHtR and categories of abnormal serum lipid indices in the two groups (both $r$ $=0.344, P<0.001)$. After adjusting for age, for men,

Table 3 Partial correlation coefficients between anthropometric indices and serum lipid levels ${ }^{\mathrm{a}}$

\begin{tabular}{|c|c|c|c|c|c|c|c|c|}
\hline & \multicolumn{2}{|c|}{$\mathrm{TG}$} & \multicolumn{2}{|c|}{ TC } & \multicolumn{2}{|c|}{ LDL-C } & \multicolumn{2}{|c|}{$\mathrm{HDL}-\mathrm{C}$} \\
\hline & $r$ & $P$ & $r$ & $P$ & $r$ & $P$ & $r$ & $P$ \\
\hline \multicolumn{9}{|l|}{$\overline{\text { Total }}$} \\
\hline WC & 0.308 & $<0.001$ & 0.189 & $<0.001$ & 0.175 & $<0.001$ & -0.347 & $<0.001$ \\
\hline BMI & 0.264 & $<0.001$ & 0.175 & $<0.001$ & 0.172 & $<0.001$ & -0.320 & $<0.001$ \\
\hline$A B S I$ & 0.152 & $<0.001$ & 0.096 & $<0.001$ & 0.080 & $<0.001$ & -0.139 & $<0.001$ \\
\hline BRI & 0.269 & $<0.001$ & 0.191 & $<0.001$ & 0.184 & $<0.001$ & -0.305 & $<0.001$ \\
\hline WHR & 0.295 & $<0.001$ & 0.168 & $<0.001$ & 0.133 & $<0.001$ & -0.291 & $<0.001$ \\
\hline WHtR & 0.278 & $<0.001$ & 0.197 & $<0.001$ & 0.189 & $<0.001$ & -0.317 & $<0.001$ \\
\hline \multicolumn{9}{|l|}{ Men } \\
\hline WC & 0.321 & $<0.001$ & 0.221 & $<0.001$ & 0.183 & $<0.001$ & -0.376 & $<0.001$ \\
\hline BMI & 0.303 & $<0.001$ & 0.209 & $<0.001$ & 0.180 & $<0.001$ & -0.367 & $<0.001$ \\
\hline ABSI & 0.153 & $<0.001$ & 0.113 & $<0.001$ & 0.074 & $<0.001$ & -0.141 & $<0.001$ \\
\hline BRI & 0.321 & $<0.001$ & 0.226 & $<0.001$ & 0.182 & $<0.001$ & -0.361 & $<0.001$ \\
\hline WHR & 0.308 & $<0.001$ & 0.209 & $<0.001$ & 0.149 & $<0.001$ & -0.305 & $<0.001$ \\
\hline $\mathrm{WH}+\mathrm{R}$ & 0.326 & $<0.001$ & 0.232 & $<0.001$ & 0.187 & $<0.001$ & -0.367 & $<0.001$ \\
\hline \multicolumn{9}{|l|}{ Women } \\
\hline WC & 0.231 & $<0.001$ & 0.131 & $<0.001$ & 0.154 & $<0.001$ & -0.282 & $<0.001$ \\
\hline BMI & 0.201 & $<0.001$ & 0.121 & $<0.001$ & 0.143 & $<0.001$ & -0.261 & $<0.001$ \\
\hline ABSI & 0.105 & $<0.001$ & 0.052 & $<0.001$ & 0.059 & $<0.001$ & -0.104 & $<0.001$ \\
\hline BRI & 0.215 & $<0.001$ & 0.126 & $<0.001$ & 0.146 & $<0.001$ & -0.259 & $<0.001$ \\
\hline WHR & 0.220 & $<0.001$ & 0.115 & $<0.001$ & 0.114 & $<0.001$ & -0.239 & $<0.001$ \\
\hline WHtR & 0.223 & $<0.001$ & 0.130 & $<0.001$ & 0.151 & $<0.001$ & -0.272 & $<0.001$ \\
\hline
\end{tabular}

WC Waist Circumference, BMI Body Mass Index, ABSI A Body Shape Index, BRI Body Roundness Index, WHR Waist-to-Hip Ratio, WHtR Waist-to-Height Ratio, TG Triglyceride, TC Total Cholesterol, LDL-C Low Density Lipoprotein Cholesterol, HDL-C High Density Lipoprotein Cholesterol 
Table 4 Spearman correlation coefficient between anthropometric indices and categories of abnormal serum lipid indices

\begin{tabular}{|c|c|c|}
\hline \multirow{3}{*}{$\overline{T o t a l}$} & \multicolumn{2}{|c|}{ Categories of abnormal serum lipid indices } \\
\hline & \multirow[t]{2}{*}{$r$} & \multirow[t]{2}{*}{$P$} \\
\hline & & \\
\hline & 0.363 & $<0.001$ \\
\hline BMI & 0.313 & $<0.001$ \\
\hline$A B S I$ & 0.229 & $<0.001$ \\
\hline BRI & 0.351 & $<0.001$ \\
\hline WHR & 0.348 & $<0.001$ \\
\hline $\mathrm{WHtR}$ & 0.351 & $<0.001$ \\
\hline \multicolumn{3}{|l|}{ Men } \\
\hline WC & 0.383 & $<0.001$ \\
\hline BMI & 0.366 & $<0.001$ \\
\hline$A B S I$ & 0.186 & $<0.001$ \\
\hline BRI & 0.377 & $<0.001$ \\
\hline WHR & 0.347 & $<0.001$ \\
\hline $\mathrm{WH} t \mathrm{R}$ & 0.377 & $<0.001$ \\
\hline \multicolumn{3}{|l|}{ Women } \\
\hline WC & 0.333 & $<0.001$ \\
\hline BMI & 0.266 & $<0.001$ \\
\hline$A B S I$ & 0.253 & $<0.001$ \\
\hline BRI & 0.344 & $<0.001$ \\
\hline WHR & 0.333 & $<0.001$ \\
\hline $\mathrm{WH}$ tR & 0.344 & $<0.001$ \\
\hline
\end{tabular}

WC Waist Circumference, BMI Body Mass Index, ABSI A Body Shape Index, BRI Body Roundness Index, WHR Waist-to-Hip Ratio, WHtR Waist-to-Height Ratio

high correlation was identified between WC and categories of abnormal serum lipid indices $(r=0.376, P<0.001)$. For women, high correlations were identified between the levels of WC, BMI, and WHtR and categories of abnormal serum lipid indices (all $r=0.239, P<0.001$ ) (Table 5).

\section{AUROC and OOPs of anthropometric indices}

We investigated AUROCs between dyslipidemia and the anthropometric indices to predict dyslipidemia. For men, AUROC value of WC was the highest among the anthropometric indices (AUROC $=0.726, P<0.001$ ). For women, AUROC values of BRI and WHtR were the highest among the anthropometric indices (both AUROC $=0.709, P<0.001)$. Men had higher OOPs of WC, BMI, ABSI, and WHR (84.050, 23.817, 0.077, and 0.880 in men, or $79.250,23.218,0.076$, and 0.847 in women). Women had higher OOPs of BRI and WHtR (3.435 and 0.504 in women, or 3.202 and 0.492 in men).

Next, we investigated AUROCs and OOPs between serum lipid levels and anthropometric indices. For men, AUROC value of WC was the highest for predicting TG abnormal level among the anthropometric indices
Table 5 Partial correlation coefficients between anthropometric indices and categories of abnormal serum lipid indices ${ }^{a}$

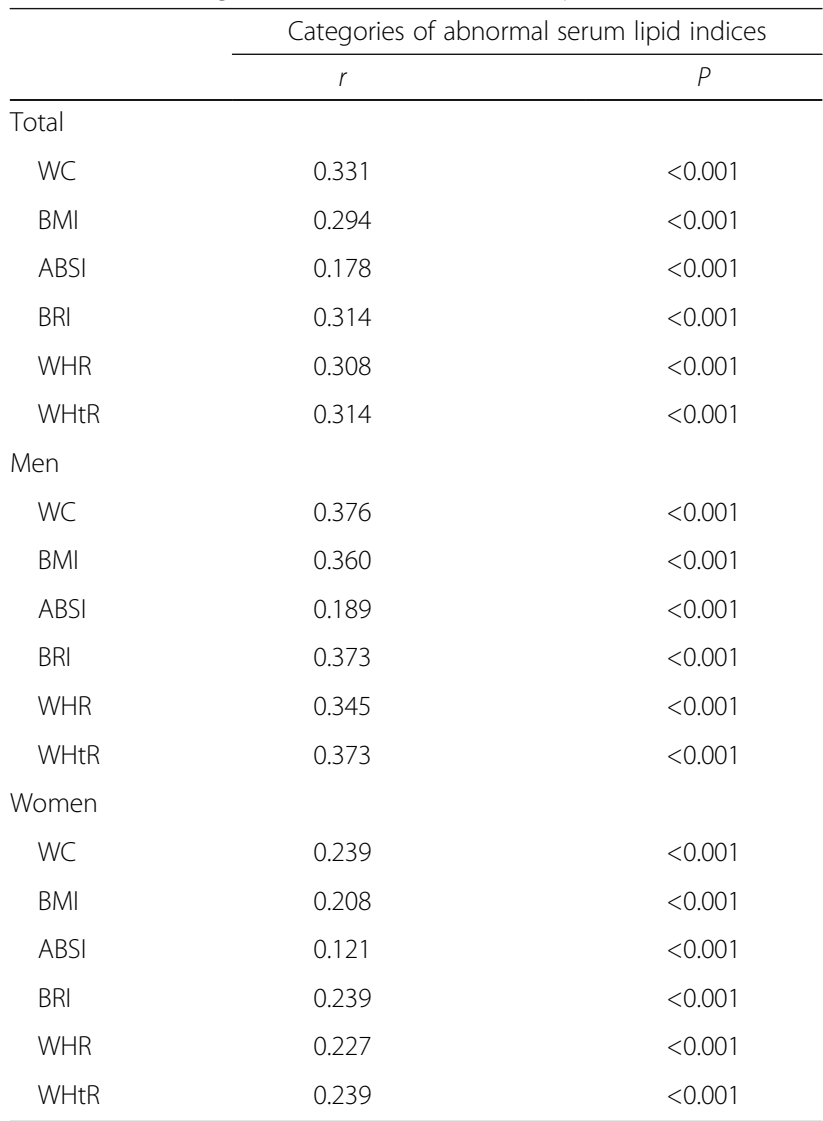

WC Waist Circumference, BMI Body Mass Index, ABSI A Body Shape Index, BRI Body Roundness Index, WHR Waist-to-Hip Ratio, WHtR Waist-to-Height Ratio

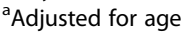

(AUROC $=0.730, \quad P<0.001$ ), and OOP of $\mathrm{WC}$ was 84.050; AUROC values of BRI and WHtR were the highest for predicting TC (both AUROC $=0.645, P<$ 0.001 ) or LDL-C abnormal levels (both AUROC $=$ $0.633, P<0.001)$ among the anthropometric indices, OOP of BRI was 3.471 for TC or 3.204 for LDL-C, and OOP of WHtR was 0.508 for TC or 0.492 for LDL-C; AUROC value of BMI was the highest for predicting HDL-C abnormal level among the anthropometric indices (AUROC $=0.695, P<0.001$ ), and OOP of BMI was 24.750. For women, AUROC values of BRI and WHtR were the highest for predicting TG (both AUROC= $0.715, P<0.001$ ), TC (both AUROC $=0.660, P<0.001$ ), or LDL-C abnormal levels (both AUROC $=0.662, P<$ 0.001) among the anthropometric indices, OOP of BRI was 3.436 for TG, 3.926 for TC, or 3.596 for LDL-C, and OOP of WHtR was 0.504 for TG, 0.512 for TC, or 0.512 for LDL-C; AUROC value of WC was the highest for predicting HDL-C abnormal level among the anthropometric indices (AUROC $=0.654, P<0.001$ ), and OOP of WC was 79.250 (Table 6, Additional file 1 : Figure S1 A-H, Table 7). 
Table 6 AUROCs for anthropometric indices and serum lipid levels

\begin{tabular}{cllll}
\hline & \multicolumn{1}{c}{ AUROC $(95 \%$ Cl) } \\
\cline { 2 - 5 } Men & \multicolumn{1}{c}{ TG } & \multicolumn{1}{c}{ LDL-C } & HDL-C \\
WC & $0.730(0.719,0.742)^{*}$ & $0.636(0.616,0.655)^{*}$ & $0.621(0.599,0.642)^{*}$ & $0.694(0.681,0.708)^{*}$ \\
BMI & $0.727(0.716,0.739)^{*}$ & $0.626(0.607,0.645)^{*}$ & $0.607(0.585,0.629)^{*}$ & $0.695(0.682,0.708)^{*}$ \\
ABSI & $0.607(0.594,0.620)^{*}$ & $0.589(0.568,0.609)^{*}$ & $0.589(0.566,0.612)^{*}$ & $0.576(0.561,0.590)^{*}$ \\
BRI & $0.728(0.717,0.739)^{*}$ & $0.645(0.626,0.664)^{*}$ & $0.633(0.611,0.654)^{*}$ & $0.683(0.669,0.696)^{*}$ \\
WHR & $0.718(0.706,0.729)^{*}$ & $0.631(0.611,0.650)^{*}$ & $0.612(0.590,0.634)^{*}$ & $0.661(0.647,0.674)^{*}$ \\
WHtR & $0.728(0.717,0.739)^{*}$ & $0.645(0.626,0.664)^{*}$ & $0.633(0.611,0.654)^{*}$ & $0.683(0.669,0.696)^{*}$ \\
Women & & & \\
WC & $0.711(0.700,0.723)^{*}$ & $0.645(0.629,0.661)^{*}$ & $0.650(0.634,0.666)^{*}$ & $0.654(0.638,0.669)^{*}$ \\
BMI & $0.672(0.660,0.684)^{*}$ & $0.601(0.583,0.618)^{*}$ & $0.613(0.596,0.631)^{*}$ & $0.627(0.612,0.643)^{*}$ \\
ABSI & $0.656(0.643,0.668)^{*}$ & $0.649(0.633,0.666)^{*}$ & $0.635(0.617,0.652)^{*}$ & $0.597(0.581,0.613)^{*}$ \\
BRI & $0.715(0.703,0.726)^{*}$ & $0.660(0.643,0.676)^{*}$ & $0.662(0.646,0.678)^{*}$ & $0.646(0.631,0.662)^{*}$ \\
WHR & $0.714(0.703,0.726)^{*}$ & $0.658(0.642,0.675)^{*}$ & $0.648(0.632,0.665)^{*}$ & $0.646(0.630,0.661)^{*}$ \\
WHtR & $0.715(0.703,0.726)^{*}$ & $0.660(0.643,0.676)^{*}$ & $0.662(0.646,0.678)^{*}$ & $0.646(0.631,0.662)^{*}$
\end{tabular}

WC Waist Circumference, BMI Body Mass Index, ABSI A Body Shape Index, BRI Body Roundness Index, WHR Waist-to-Hip Ratio, WHtR Waist-to-Height Ratio, TG Triglyceride, TC Total Cholesterol, LDL-C Low Density Lipoprotein Cholesterol, HDL-C High Density Lipoprotein Cholesterol ${ }^{*} P<0.001$

We further investigated AUROCs and OOPs between categories of abnormal serum lipid indices and anthropometric indices. For men, AUROC values of WC was the highest for predicting categories of abnormal serum lipid indices in group 1 (AUROC $=0.718, P<$ 0.001 ) or in group 2 (AUROC $=0.806, P<0.001$ ) among the anthropometric indices, OOP of WC was 82.450 for group 1 or 84.150 for group 2. For women, AUROC values of BRI and WHtR were the highest for predicting categories of abnormal serum lipid indices in group 1 (both AUROC $=0.700, P<0.001$ ) or in group 2 (both AUROC $=0.783, P<0.001)$ among the anthropometric indices, OOP of BRI was 3.435 for group 1 or 3.926 for group 2, and OOP of WHtR was 0.504 for group 1 or 0.529 for group 2 (Table 8, Additional file 1: Figure S1 I-L, Table 9).

Table 7 Optimal operating points of anthropometric indices for predicting abnormal serum lipid levels

\begin{tabular}{|c|c|c|c|c|c|c|c|c|c|c|c|c|}
\hline & \multicolumn{3}{|c|}{$\mathrm{TG}$} & \multicolumn{3}{|c|}{ TC } & \multicolumn{3}{|c|}{ LDL-C } & \multicolumn{3}{|c|}{$\mathrm{HDL}-\mathrm{C}$} \\
\hline & $\mathrm{OOP}$ & SEN(\%) & SPE(\%) & $\mathrm{OOP}$ & SEN(\%) & SPE(\%) & $\mathrm{OOP}$ & SEN(\%) & SPE(\%) & OOP & SEN(\%) & SPE $(\%)$ \\
\hline \multicolumn{13}{|l|}{ Men } \\
\hline WC & 84.050 & 76.015 & 60.097 & 84.350 & 69.829 & 51.318 & 80.750 & 80.789 & 37.242 & 85.350 & 70.412 & 59.265 \\
\hline BMI & 24.512 & 70.269 & 65.015 & 23.817 & 71.805 & 48.436 & 23.543 & 72.213 & 44.805 & 24.750 & 67.024 & 63.291 \\
\hline$A B S I$ & 0.077 & 69.225 & 47.612 & 0.077 & 69.170 & 45.581 & 0.078 & 57.804 & 55.556 & 0.077 & 69.283 & 43.383 \\
\hline BRI & 3.253 & 78.224 & 57.324 & 3.471 & 67.721 & 55.061 & 3.204 & 75.129 & 46.285 & 3.301 & 73.574 & 53.527 \\
\hline WHR & 0.881 & 76.215 & 56.967 & 0.897 & 62.187 & 57.861 & 0.863 & 79.760 & 37.762 & 0.890 & 67.871 & 57.458 \\
\hline $\mathrm{WH} t \mathrm{R}$ & 0.492 & 79.911 & 55.607 & 0.508 & 66.930 & 55.785 & 0.492 & 75.129 & 46.259 & 0.497 & 73.518 & 53.574 \\
\hline \multicolumn{13}{|l|}{ Women } \\
\hline WC & 81.250 & 70.699 & 62.335 & 80.450 & 67.262 & 54.108 & 79.550 & 72.527 & 50.325 & 79.250 & 73.697 & 50.590 \\
\hline BMI & 22.987 & 81.106 & 45.580 & 23.327 & 70.273 & 44.977 & 23.500 & 70.030 & 47.114 & 24.363 & 63.192 & 57.032 \\
\hline$A B S I$ & 0.077 & 69.808 & 54.924 & 0.077 & 65.193 & 57.674 & 0.076 & 71.029 & 49.380 & 0.076 & 64.475 & 50.833 \\
\hline BRI & 3.436 & 78.856 & 54.597 & 3.926 & 59.831 & 53.953 & 3.596 & 69.930 & 54.196 & 3.435 & 72.975 & 50.067 \\
\hline WHR & 0.862 & 66.526 & 65.059 & 0.829 & 80.903 & 42.088 & 0.831 & 79.520 & 42.925 & 0.861 & 60.946 & 60.423 \\
\hline $\mathrm{WHtR}$ & 0.504 & 78.762 & 54.652 & 0.512 & 69.332 & 53.953 & 0.512 & 70.230 & 53.889 & 0.504 & 72.975 & 50.067 \\
\hline
\end{tabular}

WC Waist Circumference, BMI Body Mass Index, ABSI A Body Shape Index, BRI Body Roundness Index, WHR Waist-to-Hip Ratio, WHtR Waist-to-Height Ratio, TG Triglyceride, TC Total Cholesterol, LDL-C Low Density Lipoprotein Cholesterol, HDL-C High Density Lipoprotein Cholesterol, OOP Optimal Operating Points, SEN Sensitivity; SPE: Specificity 
Table 8 AUROCs for anthropometric indices and categories of abnormal serum lipid indices

\begin{tabular}{ccc}
\hline & \multicolumn{2}{c}{ AUROC(95\%Cl) } \\
\cline { 2 - 3 } Men & \multicolumn{2}{c}{ One/two } \\
WC & $0.718(0.707,0.730)^{*}$ & $0.806(0.785,0.826)^{*}$ \\
BMI & $0.712(0.701,0.724)^{*}$ & $0.793(0.770,0.815)^{*}$ \\
ABSI & $0.603(0.591,0.616)^{*}$ & $0.663(0.635,0.690)^{*}$ \\
BRI & $0.714(0.703,0.726)^{*}$ & $0.805(0.784,0.826)^{*}$ \\
WHR & $0.702(0.691,0.714)^{*}$ & $0.768(0.745,0.792)^{*}$ \\
WHtR & $0.714(0.703,0.726)^{*}$ & $0.805(0.784,0.826)^{*}$ \\
Women & & \\
WC & $0.697(0.686,0.708)^{*}$ & $0.773(0.752,0.794)^{*}$ \\
BMI & $0.660(0.648,0.671)^{*}$ & $0.713(0.688,0.737)^{*}$ \\
ABSI & $0.648(0.637,0.660)^{*}$ & $0.721(0.696,0.746)^{*}$ \\
BRI & $0.700(0.689,0.711)^{*}$ & $0.783(0.762,0.804)^{*}$ \\
WHR & $0.696(0.685,0.708)^{*}$ & $0.774(0.752,0.796)^{*}$ \\
WHtR & $0.700(0.689,0.711)^{*}$ & $0.783(0.762,0.804)^{*}$ \\
\hline WC Waist Circumference, BMI Body Mass Index, ABSI A Body Shape Index, BRI \\
Body Roundness Index, WHR Waist-to-Hip Ratio, WHtR Waist-to-Height Ratio \\
*P $<0.001$
\end{tabular}

\section{Discussion}

This study mainly focusses on identifying the capacity of commonly used anthropometric indices (WC, BMI, ABSI, BRI, WHR, and WHtR) in prediction for dyslipidemia. Our results showed that all the anthropometric indices

Table 9 Optimal operating points of anthropometric indices for predicting categories of abnormal serum lipid indices

\begin{tabular}{cccccccc}
\hline & \multicolumn{3}{c}{ One/two } & & \multicolumn{3}{c}{ Three/more } \\
& OOP & SEN(\%) & SPE(\%) & OOP & SEN(\%) & SPE(\%) \\
\hline Men & & & & & & & \\
WC & 82.450 & 75.462 & 57.737 & 84.150 & 84.640 & 64.224 \\
BMI & 23.877 & 70.129 & 61.789 & 24.787 & 77.116 & 71.441 \\
ABSI & 0.077 & 66.523 & 50.055 & & 0.076 & 82.759 & 42.993 \\
BRI & 3.202 & 73.274 & 59.752 & & 3.516 & 80.878 & 68.408 \\
WHR & 0.880 & 71.270 & 59.863 & & 0.897 & 73.041 & 69.648 \\
WHtR & 0.492 & 73.274 & 59.752 & & 0.508 & 80.878 & 68.408 \\
Women & & & & & & \\
WC & 79.250 & 71.438 & 58.582 & & 80.750 & 80.217 & 63.243 \\
BMI & 23.218 & 73.863 & 51.313 & & 24.216 & 70.732 & 61.913 \\
ABSI & 0.076 & 70.309 & 52.855 & & 0.077 & 73.984 & 59.928 \\
BRI & 3.435 & 72.434 & 58.500 & & 3.926 & 72.629 & 71.529 \\
WHR & 0.842 & 71.073 & 57.434 & & 0.863 & 72.358 & 68.608 \\
WHtR & 0.504 & 72.434 & 58.500 & 0.529 & 72.629 & 71.529 \\
\hline
\end{tabular}

WC Waist Circumference, BMI Body Mass Index, ABSI A Body Shape Index, BRI Body Roundness Index, WHR Waist-to-Hip Ratio, WHtR Waist-to-Height Ratio, OOP Optimal Operating Points, SEN Sensitivity, SPE Specificity can predict dyslipidemia independently because they all had AUROCs $>0.5$.

Anthropometric indices (BMI, WC, WHR, WHtR, and ABSI) have positive correlation with TG, TC, and LDL-C levels, but have negative correlation with HDL-C level [36-39]. BRI positively correlates with TG and LDL-C levels, but negatively correlates with HDL-C level [40]. Our results also supported those previous results. Moreover, we identified that BRI also had positive correlation with TC levels.

Age-specific BMI and WC are associated with CVD risk factors, including fasting insulin, TG, HDL-C, and LDL-C among Chinese children [41]. A cohort study, which included 8940 Chinese adults, reveals that BMI is strongly associated with hypertension, and BMI has higher AUROC and prevalence ratio, while WC is associated with diabetes and dyslipidemia [42]. Age-specific WC is superior to BMI in predicting MetS in children [43]. Working Group on Obesity in China has also recommended that BMI is a better predictor of hypertension in adults, while WHtR and WC are more sensitive to predict diabetes and dyslipidemia [44].

In this study, we furthermore identified that WC, BMI, and WHtR were the best predictors for TG, HDL-C, and TC or LDL-C abnormal levels in men respectively; WHtR was the best predictor for TG, TC, or LDL-C abnormal levels, and WC was the best predictor for HDL-C abnormal level in women.

ABSI could be served as a substantial risk factor for premature mortality in Korean population [45]. However, ABSI has no evidence to distinguish between individuals with and without CVD or CVD risk factors, including dyslipidemia [46]. Compared with BMI and WC, ABSI has similar predictive ability for initial stage diabetes in a prospective cohort study in China which includes 687 people after a follow up of 15 years, indicating that ABSI in this respect is not superior to BMI or WC [47]. ABSI is not the best predictor of hypertension, diabetes, and dyslipidemia for Japanese adults in retrospective cohort of 48,953 Japanese adults during a follow-up of 4 years [30]. Our results supported those above results. Our results validated ABSI could not be used as the best sensitive predictor for abnormal TG, TC, LDL-C, and HDL-C levels respectively. In contrast, Haghighatdoost et al. have shown the highest odds ratio was observed for ABSI and MetS in different age and sex categories in a population-based cohort of 9555 Iranian adults aged $\geq 19$ years [48]. The endpoint of variable choices [46] and the weak correlation between ABSI and height [48] may contribute to the discrepancy.

BRI has a good discriminative ability for either diabetes or CVD and its risk factors, and BRI has a larger AUROC value than BMI and WC $[31,46,49]$. BRI is superior to WHtR: BRI can accurately estimate the 
percentage of body fat and visceral adipose tissues [28]. In our study, BRI levels in men were different from those in women. For men, BRI was not the best predictor for dyslipidemia; however, for women, BRI was the best predictor for dyslipidemia. We found BRI could be used as the best sensitive predictor for TG abnormal level in women and TC or LDL-C abnormal level in men and women respectively.

To our knowledge, this is the first study to investigate relationship between the anthropometric indices and categories of abnormal serum lipid indices. Our results demonstrated WC could be used to identify all categories of abnormal serum lipid indices in men; WHtR and BRI could be used to identify all categories of abnormal serum lipid indices in women. Moreover, our results further revealed that the values of anthropometric indices (BMI, WC, WHR, WHtR, and BRI) increase with increasing categories of abnormal serum lipid indices in both men and women groups.

This study has limitations. First, pharmacological treatments, diets, and nutraceuticals can influence results. Second, ABSI was developed to predict mortality hazard in a follow-up study; however, we used ABSI to predict dyslipidemia in a cross-sectional study. Third, the sensitivity of BRI to determine risk of dyslipidemia in a clinical setting remains elusive.

\section{Conclusions}

In our study, WC was a good predictor for one/two or three/more categories of abnormal serum lipid indices in men. However, BRI and WHtR were good predictors for one/two or three/more categories of abnormal serum lipid indices in women. ABSI showed the weakest predictive power. These indices are necessary for screening of dyslipidemia in clinical practice.

\section{Additional file}

Additional file 1: Receiver Operating Characteristic (ROC) curves for anthropometric indices and serum lipid levels. (DOCX $334 \mathrm{~kb}$ )

\section{Abbreviations}

ABSI: A Body shape index; AUROC: Area under the receiver operating characteristic curve; BMI: Body mass index; BRI: Body roundness index; CVD: Cardiovascular disease; HDL-C: High-density cholesterol; LDL-C: Lowdensity cholesterol; MetS: Metabolic syndrome; OOP: Optimal operating point; ROC: Receiver operating characteristic; SEN: Sensitivity; SPE: Specificity; TC: Total cholesterol; TGs: Triglycerides; WC: Waist circumference; WHR: Waistto-hip ratio; WHtR: Waist-to-height ratio

\section{Acknowledgements}

We thank all teachers and students as investigators who participated in this study. We acknowledge support from Health Bureau of Jilin Province, China.

\section{Funding}

This work was funded by the National Natural Science Foundation of China (grant number: 81573230), the Science Technology Department of Jilin

Province (grant number: $20150101130 J C)$, the Scientific Research Foundation of the Health Bureau of Jilin Province, China (grant number: 2011Z116), and the Ministry of Science and Technology of the People's Republic of China (grant number: 2015DFA31580).

\section{Availability of data and materials}

The data that support the findings of this study are available from School of Public Health, Jilin University but restrictions apply to the availability of these data, which were used under license for the current study, and so are not publicly available. Data are however available from the authors upon reasonable request and with permission of School of Public Health, Jilin University.

\section{Authors' contributions}

Kaixin Zhang contributed to data analysis, drafting the manuscript and bears the primary responsibility for the content of the manuscript. Qian Zhao was involved in revision of the manuscript. Yong Li, Qing Zhen, Yaqin Yu, and Yuchun Tao collected the data. Yi Cheng was involved in the revision and polish of the manuscript. Yawen Liu contributed to conception and design. All the authors read and approved the content of the manuscript.

\section{Ethics approval and consent to participate}

The study was approved by the Ethics Committee of Jilin University, and all participants in the study provided signed informed consents.

\section{Consent for publication}

Not applicable.

\section{Competing interests}

The authors declare that they have no competing interest.

\section{Publisher's Note}

Springer Nature remains neutral with regard to jurisdictional claims in published maps and institutional affiliations.

\section{Author details}

'Department of Epidemiology and Biostatistics, School of Public Health, Jilin University, Changchun 130021, China. ${ }^{2}$ The Cardiovascular Center, The First Hospital of Jilin University, Changchun 130021, China.

Received: 6 November 2017 Accepted: 21 December 2017

Published online: 22 January 2018

\section{References}

1. Joshi SR, Anjana RM, Deepa M, Pradeepa R, Bhansali A, Dhandania VK, et al. Prevalence of Dyslipidemia inurban and rural india: the ICMR-INDIAB study. PLoS One. 2014;9(5):e96808. https://doi.org/10.1371/journal.pone.0096808.

2. Musunuru K. Atherogenic Dyslipidemia: cardiovascular risk and dietary intervention[J]. Lipids. 2010;45(10):907-14. https://doi.org/10.1007/s11745010-3408-1.

3. Haffner SM. Diabetes, hyperlipidemia, and coronary artery disease. Am J Cardiol. 1999:83(9B):17F-21F.

4. Sea S. Urbanization and cardiovascular disease: raising heart-healthy children in today's cities. Geneva: The World Heart Federation; 2012

5. Gidding SS. A perspective on obesity. Am J Med Sci. 1995;310(Suppl 1): S68-71.

6. World Health Organization. Obesity: preventing and managing the global epidemic. Report: Geneva, World Health Organization; 1997.

7. World Health Organization. Physical status: the use and interpretation of anthropometry: report of a WHO expert committee. World Health Organ Tech Rep Ser. 1995:854:1-452.

8. Cai L, Liu A, Zhang Y, Wang P. Waist-to-height ratio and cardiovascular risk factors among Chinese adults in Beijing. PLoS One. 2013;8(7):e69298. https://doi.org/10.1371/journal.pone.0069298.

9. WHO. Obesity: preventing and managing the global epidemic. Report of a World Health Organization consultation. WHO Tech Rep Ser. 2000;894(i-xii): $1-253$.

10. Daniels SR, Khoury PR, Morrison JA. Utility of different measures of body fat distribution in children and adolescents. Am J Epidemiol. 2000;152(12): 1179-84.

11. Li C, Ford ES, McGuire LC, Mokdad AH. Increasing trends in waist circumference and abdominal obesity among US adults. Obesity. 2007;15(1): 216-24. https://doi.org/10.1038/oby.2007.505. 
12. Esmaillzadeh A, Mirmiran P, Azizi F. Waist-to-hip ratio is a better screening measure for cardiovascular risk actors than other anthropometric indicators in Tehranian adult men. Int J Obes Relat Metab Disord. 2004;28(10):1325-32. https://doi.org/10.1038/sj.ijo.0802757.

13. Schneider HJ, Glaesmer H, Klotsche J, Bohler S, Lehnert H, Marz W, et al. Accuracy of anthropometric indicators of obesity to predict cardiovascular risk. J Clin Endocrinol Metab. 2007;92(2):589-94. https://doi.org/10.1210/jc. 2006-0254.

14. Caan B, Armstrong MA, Selby JV, Sadler M, Folsom AR, Jacobs D, et al. Changes in measurements of body fat distribution accompanying weight change. Int J Obes Relat Metab Disord. 1994;18(6):397-404.

15. Cornier MA, Despres JP, Davis N, Grossniklaus DA, Klein S, Lamarche B, et al. Assessing adiposity: a scientific statement from the American Heart Association. Circulation. 2011;124(18):1996-2019. https://doi.org/10.1161/CIR. 0b013e318233bc6a.

16. Nyamdorj R, Qiao Q, Lam TH, Tuomilehto J, Ho SY, Pitkaniemi J, et al. BMI compared with central obesity indicators in relation to diabetes and hypertension in Asians. Obesity (Silver Spring). 2008;16(7):1622-35. https:// doi.org/10.1038/oby.2008.73.

17. InterAct C, Langenberg C, Sharp SJ, Schulze MB, Rolandsson O, Overved K, et al. Long-term risk of incident type 2 diabetes and measures of overall and regional obesity: the EPIC-InterAct casecohort study. PLoS Med. 2012; 9(6):e1001230. https://doi.org/10.1371/journal.pmed.1001230.

18. Hsieh S, Yoshinaga H, Muto T. Waist-to-height ratio, a simple and practical index for assessing central fat distribution and metabolic risk in Japanese men and women. Int J Obes Relat Metab Disord. 2003;27(5):610-6. https:// doi.org/10.1038/sj.ijo.0802259.

19. He Y, Zhai F, Ma G, Feskens E, Zhang J, Fu P, et al. Abdominal obesity and the prevalence of diabetes and intermediate hyperglycaemia in Chinese adults. Public Health Nutr. 2009:12(8):1078-84. https://doi.org/10.1017/ S1368980008003856.

20. Ashwell M, Lejeune S, McPherson K. Ratio of waist circumference to height may be better indicator of need for weight management. BMJ. 1996:312-77.

21. Lin WY, Lee LT, Chen CY, Lo H, Hsia HH, Lin RS, et al. Optimal cut-off values for obesity: using simple anthropometric indices to predict cardiovascular risk factors in Taiwan. Int J Obes Relat Metab Disord. 2002;26(9):1232-8. https://doi.org/10.1038/sj.ijo.0802040.

22. Shao J, Yu L, Shen X, Li D, Wang K. Waist-to-height ratio, an optimal predictor for obesity and metabolic syndrome in Chinese adults. J Nutr Health Aging. 2010;14(9):782-5.

23. Ho SY, Lam TH, Janus ED. Waist to stature ratio is more strongly associated with cardiovascular risk factors than other simple anthropometric indices. Ann Epidemiol. 2003;13(10):683-91.

24. Ashwell M, Gibson S. Waist to height ratio is a simple and effective obesityscreening tool for cardiovascular risk factors: analysis of data from the BritishNational diet and nutrition survey of adults aged 19-64 years. Obes Facts. 2009;2(2):97-103. https://doi.org/10.1159/000203363.

25. Ashwell M, Gunn P, Gibson S. Waist-to-height ratio is a better screening tool than waist circumference and BMI for adult cardiometabolic risk factors: systematic review and meta-analysis. Obes Rev. 2012;13(3):275-86. https:// doi.org/10.1111/j.1467-789X.2011.00952.x.

26. Lee CM, Huxley RR, Wildman RP, Woodward M. Indices of abdominalobesity are better discriminators of cardiovascular risk factors than BMl: a metaanalysis. J Clin Epidemiol. 2008;61(7):646-53. https://doi.org/10.1016/j. jclinepi.2007.08.012.

27. Krakauer NY, Krakauer JC. A new body shape index predicts mortality hazard independently of body mass index. PLoS One. 2012;7(7):e39504. https://doi. org/10.1371/journal.pone.0039504.

28. Thomas DM, Bredlau C, Bosy-Westphal A, Mueller M, Shen W, Gallagher D, et al. Relationships between body roundness with body fat and visceral adipose tissue emerging from a new geometrical model. Obesity. 2013; 21(11):2264-71. https://doi.org/10.1002/oby.20408.

29. Krakauer NY, Krakauer JC. Dynamic association of mortality hazard with body shape. PLoS One. 2014;9:e88793. https://doi.org/10.1371/journal.pone. 0088793.

30. Fujita M, Sato Y, Nagashima K, Takahashi S, Hata A. Predictive power of a body shape index for development of diabetes, hypertension, and dyslipidemia in Japanese adults: a retrospective cohort study. PLoS One. 2015;10:e0128972. https://doi.org/10.1371/journal.pone.0128972.

31. Chang Y, Guo X, Li T, Guo J, Sun Y. A body shape index and body roundness index: two new body indices to identify left ventricular hypertrophy among rural populations in northeast China. Heart Lung Circ 2016:25:358 364. https://doi.org/10.1016/j.hlc.2015.08.009.

32. Expert Consultation WHO. Appropriate body-mass index for Asian populations and its implications for policy and intervention strategies. Lancet. 2004;363:157-63.

33. Garrow JS. Quetelet index as indicator of obesity. Lancet. 1986;327(8491): 1219.

34. Adult Dyslipidemia Prevention Guide of China. People's health publishing house. China: Beijing; 2007.

35. Chen FY, Xue YQ, Tan MT, Chen PY. Efficient statistical tests to compare Youden index: accounting for contingency correlation. Stat Med. 2015;34(9): 1560-76. https://doi.org/10.1002/sim.6432.

36. Gu D, Reynolds K, Wu X, Chen J, Duan X, Reynolds RF, et al. Prevalence of the metabolic syndrome and overweight among adults in China. Lancet. 2005:365:1398-405.

37. Girotto E, Andrade SM, Cabrera MA. Prevalence of abdominal obesity in hypertensive patients registered in a family health unit. Arq Bras Cardiol. 2010;94(6):754-62.

38. Weiler Miralles CS, Wollinger LM, Marin D, Genro JP, Contini V, Da M, Bosco S. Waist-to-height ratio (WHtR) and triglyceride to $\mathrm{HDL}-\mathrm{c}$ ratio $(\mathrm{TG} / \mathrm{HDL}-\mathrm{c})$ as predictors of cardiometabolic risk. Nutr Hosp. 2015;31(5):2115-21. https:// doi.org/10.3305/nh.2015.31.5.7773.

39. Malara M, Keska A, Tkaczyk J, Lutosławska G. Body shape index versus body mass index as correlates of health risk in young healthy sedentary men. J Transl Med. 2015;13:75. https://doi.org/10.1186/s12967-015-0426-z.

40. Zaid M, Ameer F, Munir R, Rashid R, Farooq N, Hasnain S, et al. Anthropometric and metabolic indices in assessment of type and severity of dyslipidemia. J Physiol Anthropol. 2017;36(1):19. https://doi.org/10.1186/ s40101-017-0134-x.

41. Sung RY, CC Y, Choi KC, McManus A, Li AM, SL X, et al. Waist circumference and body mass index in Chinese children: cut-off values for predicting cardiovascular risk factors. Int J Obes. 2007;31:550-8.

42. Feng RN, Zhao C, Wang C, Niu YC, Li K, Guo FC, et al. BMl is strongly associated with hypertension, and waist circumference is strongly associated with type 2 diabetes and dyslipidemia, in northern Chinese adults. J Epidemiol. 2012;22:317-23.

43. Moreno LA, Pineda I, Rodriguez G, Fleta J, Sarria A, Bueno M. Waist circumference for the screening of the metabolic syndrome in children. Acta Paediatr. 2002;91:1307-12.

44. Bei-Fan Z. Cooperative meta-analysis Group of Working Group on obesity in China predictive values of body mass index and waist circumference for risk factors of certain related diseases in Chinese adults: study on optimal cutoff points of body mass index and waist circumference in Chinese adults. Asia Pac J Clin Nutr. 2002:11:S685-93.

45. Kang SM, Yoon JW, Ahn HY, Kim SY, Lee KH, Shin H, et al. Android fat depot is more closely associated with metabolic syndrome than abdominal visceral fat in elderly people. PLoS One. 2011;6:e27694. https://doi.org/10. 1371/journal.pone.0027694.

46. Maessen MF, Eijsvogels TM, Verheggen RJ, Hopman MT, Verbeek AL, F d V. Entering a new era of body indices: the feasibility of a body shape index and body roundness index to identify cardiovascular health status. PLoS One. 2014;9:e107212. https://doi.org/10.1371/journal.pone.0107212.

47. He S, Chen X. Could the new body shape index predict the new onset of diabetes mellitus in the Chinese population? PLoS One. 2013;8:e50573. https://doi.org/10.1371/journal.pone.0050573.

48. Haghighatdoost F, Sarrafzadegan N, Mohammadifard N, Asgary S, Boshtam $M$, Azadbakht L. Assessing body shape index as a risk predictor for cardiovascular diseases and metabolic syndrome among Iranian adults. Nutrition. 2014;30:636-44. https://doi.org/10.1016/j.nut.2013.10.021.

49. Tian S, Zhang $X, X u Y$, Dong $H$. Feasibility of body roundness index for identifying a clustering of cardiometabolic abnormalities compared to BMI, waist circumference and other anthropometric indices: the China health and nutrition survey, 2008 to 2009. Medicine. 2016;95(34):e4642. https://doi. org/10.1097/MD.0000000000004642. 